See discussions, stats, and author profiles for this publication at: https://www.researchgate.net/publication/301599687

\title{
Seminal plasma arising from the whole boar sperm-rich fraction increases the stability of sperm membrane after thawing
}

Article in Journal of Animal Science · May 2016

DOI: 10.2527/jas.2016-0293

CITATIONS

4

11 authors, including:

Mariana Andrade Torres

University of São Paulo

67 PUBLICATIONS 41 CITATIONS

SEE PROFILE

Simone Maria Massami Kitamura Martins

University of São Paulo

74 PUBLICATIONS 133 CITATIONS

SEE PROFILE

Some of the authors of this publication are also working on these related projects:

Genetic Modification of Livestock View project

Influence of seminal plasma from the rich fraction of boar ejaculate on structural characteristics, kinetics, capacitation and fertility of the swine spermatozoa stored at $17^{\circ} \mathrm{C}$ for 72 hours View project

\section{READS}

134

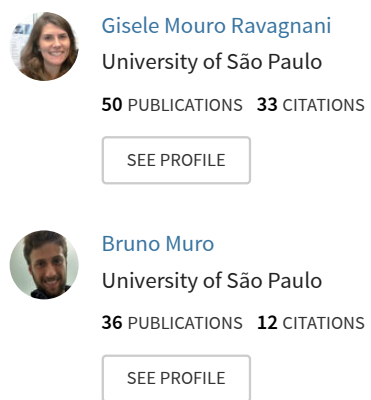




\title{
Seminal plasma arising from the whole boar sperm-rich fraction increases the stability of sperm membrane after thawing 1,2
}

\author{
M. A. Torres, * G. M. Ravagnani, * D. F. Leal,* S. M. M. K. Martins, *† B. B. D. Muro, * \\ F. V. Meirelles, $\$$ F. O. Papa,§ J. A. Dell'aqua Junior,§ M. A. Alvarenga, § A. S. Moretti, $\dagger$ \\ and A. F. C. De Andrade*3
}

*Laboratory of Andrology and Technology of Swine Embryos, Department of Animal Reproduction, School of Veterinary Medicine and Animal Science, University of São Paulo, Pirassununga, São Paulo, Brazil, 13635-900; $\dagger$ Laboratory of Swine Research, Department of Animal Nutrition and Production, School of Veterinary Medicine and Animal Science, University of São Paulo, Pirassununga, São Paulo, Brazil, 13635-900; \$Department of Veterinary Medicine, School of Animal Sciences and Food Engineering, University of São Paulo, Pirassununga, São Paulo, Brazil 13635-900; and §Department of Animal Reproduction and Veterinary Radiology, School of Veterinary Medicine and Animal Science, São Paulo State University (UNESP), Botucatu, São Paulo, Brazil, 18618-970

\begin{abstract}
Boar spermatozoa arising from the sperm-rich ejaculate fraction are reported to have a more stable plasma membrane and are more resistant to cold shock and premature acrosome reaction than spermatozoa from the whole ejaculate. Furthermore, seminal plasma (SP) can increase the cryotolerance of boar spermatozoa, and in other domestic species, it has the ability to reverse cryopreservation damage. This study aimed to evaluate the effects of boar SP arising from the whole sperm-rich ejaculate fraction (SP-SRF) on the integrity, stability, and peroxidation of sperm membranes after thawing. Each ejaculate ( $n$ $=24$ ) was divided among 4 treatments: control (CT), centrifuged and suspended in autologous SP-SRF (CS), centrifuged with withdrawn SP-SRF (CW), and post-thawed SP arising from the whole sperm-rich
\end{abstract}

fraction addition to CW (CWSP). After thawing, all treatments were incubated for 5, 60, and $120 \mathrm{~min}$ and were analyzed for membrane integrity, fluidity, and peroxidation by flow cytometer. The absence of SPSRF increased the lipid disorder $(P<0.05)$ but had no effect on lipid peroxidation $(P>0.05)$ or membrane integrity $(P>0.05)$. However, the increase in lipid disorder by withdrawal of SP-SRF was reversed by SP-SRF addition $(P<0.05)$ to the post-thawing medium, whereas plasma and acrosomal membrane integrity $(P>0.05)$ and lipid peroxidation $(P>0.05)$ were unchanged. In conclusion, despite the centrifugation effects, the addition of SP arising from the whole sperm-rich fraction to post-thawed boar semen decreased sperm lipid disorder without an influence of the sperm membrane integrity and peroxidation.

Key words: lipid disorder, lipid peroxidation, membrane integrity, seminal plasma, spermatozoa, swine

(C) 2016 American Society of Animal Science. All rights reserved. J. Anim. Sci. 2016.94:1906-1912
doi:10.2527/jas2016-0293

\footnotetext{
${ }^{1}$ This research was supported by Fundação de Amparo à Pesquisa do Estado de São Paulo (FAPESP grants 2011/23484-8 and 2013/08070-8). Extenders were donated by Botupharma, and Agroceres Multimix assisted with the nutritional support.

${ }^{2}$ M. A. Torres, G. M. Ravagnani, D. F. Leal, S. M. M. K. Martins, B. B. D. Muro, F. V. Meirelles, F. O. Papa, J. A. Dell'aqua Junior, M. A. Alvarenga, A. S. Moretti, and A. F. C. De Andrade report no conflict of interest.

${ }^{3}$ Corresponding author: andrefc@usp.br

Received January 11, 2016.

Accepted March 7, 2016.
}

\section{INTRODUCTION}

Seminal plasma (SP) has been used during cryopreservation in various domestic species, such as bulls (Garner et al., 2001), stallions (de Andrade et al., 2012), rams (Rovegno et al., 2013), and boars (Fernández-Gago et al., 2013), due to its protective effect against cold shock (Pursel et al., 1973). However, despite mounting evidence that SP exerts an important role in spermatic
\end{abstract}


cell physiology (Caballero et al., 2012), its effects within and between different species remain controversial.

Alternatively, the use of specific fractions of boar ejaculate, rather than the whole ejaculate, has been broadly studied with promising results (Saravia et al., 2007). Spermatozoa arising from the sperm-peak portion (P1; the first $10 \mathrm{~mL}$ of the sperm-rich fraction [SRF]) are more resistant to cold shock and the premature acrosome reaction and have a more stable plasma membrane (Saravia et al., 2009), resulting in an increased post-thaw survival rate (Saravia et al., 2009). However, the P1 represents only half of the spermatozoa in the SRF, therefore wasting spermatozoa in the other parts of the SRF (Zasiadczyk et al., 2015). Notwithstanding, use of only the $\mathrm{P} 1$ results in a huge loss of spermatozoa, which cannot be compensated by its benefits.

The use of whole SRF can be an alternative to the use of only the P1. It has a great seminal protein concentration, since SRF has a richer vesicular gland protein secretion than the $\mathrm{P} 1$, that practically has only epididymal caudal fluid content (Siqueira et al., 2011). So the sperm-peak or whole SRF can be used as an additive in sperm preservation technologies compared with the post-SRF (García et al., 2009).

Therefore, we sought 1) if it is possible to minimize/reverse the capacitation-like changes in frozenthawed boar semen using the SP-SRF added after thawing, 2) whether the addition or maintenance of SP-SRF is capable of minimizing the harmful cryopreservation effects in regards to increasing lipid peroxidation and the integrity of plasma and the acrosomal membrane, and 3) whether centrifugation has an effect on the post-thawed boar semen quality, enabling separation of the centrifugation and the SP effects.

\section{MATERIALS AND METHODS}

All animal procedures were performed according to the legal and ethical standards of the Ethics Committee for the use of Animals of the School of Veterinary Medicine and Animal Science of the University of São Paulo under protocol 3066/2013.

\section{Reagents and Chemicals}

Semen extender medium (Botu-Sui) was purchased from Biotech Botucatu Ltd./ME (Botucatu, SP, Brazil). The fluorescent probes Hoechst 33342 (H342), C11-BODIPY581/591, Yo-Pro-1, and Merocyanine 540 (M540) were purchased from Molecular Probes (Eugene, OR). Unless otherwise stated, all other chemicals were purchased from Sigma-Aldrich (St. Louis, MO).

\section{Staining Incubation Media}

Semen samples were diluted in Tyrode's albumin lactate pyruvate (TALP) sperm medium (Bavister et al., 1983) or a modified TALP (mTALP; $114 \mathrm{mM} \mathrm{NaCl}, 3.2$ $\mathrm{m} M \mathrm{KCl}, 0.5 \mathrm{~m} M \mathrm{MgCl}_{2} \cdot 6 \mathrm{H} 2 \mathrm{O}, 0.4 \mathrm{~m} M \mathrm{NaH}_{2} \mathrm{PO}_{4} \cdot \mathrm{H}_{2} \mathrm{O}$, $5 \mathrm{~m} M$ glucose, $10 \mathrm{~m} M$ sodium lactate, $0.1 \mathrm{~m} M$ sodium pyruvate, and 10,000 IU/100 mL sodium penicillin without sodium bicarbonate). The medium $\mathrm{pH}$ was adjusted to 7.4 using $5 \mathrm{~N} \mathrm{NaOH}$. During staining, TALP medium was maintained at $37^{\circ} \mathrm{C}$ in a water bath.

\section{Semen Collection, Raw Semen Evaluation, and Freezing}

Four whole SRF were obtained from each of 6 boars $(n=24)$ by the gloved-hand technique. Semen was distributed into 3 treatments as follows: control (CT), centrifuged and suspended in autologous SPSRF (CS; the pellet was gently mixed in its own supernatant, without SP-SRF withdraw), and centrifuged with withdrawn SP $(\mathbf{C W})$. The CS pellet was suspended in its own SP-SRF after centrifugation $(500 \times g$ for 10 min at $25^{\circ} \mathrm{C}$. Treatment $\mathrm{CW}$ was centrifuged $(500 \times \mathrm{g}$ for $10 \mathrm{~min}$ at $25^{\circ} \mathrm{C}$ ), and the supernatant was separated from the pellet and preserved (see the next section). The $\mathrm{CT}$, CS, and pellet obtained for CW were suspended with freezing extender to obtain a final concentration of $300 \times 10^{6}$ spermatozoa/mL and were stored in $0.5-\mathrm{mL}$ straws (IMV Technologies, Laigle, France). The straws were subsequently placed onto an automatic freezing system (TK 3000; TK Tecnologia em Congelação Ltda, Uberaba, Brazil) and cooled at a rate of $-0.5^{\circ} \mathrm{C} / \mathrm{min}$ to $5^{\circ} \mathrm{C}$. The freezing rate used was $-20^{\circ} \mathrm{C} / \mathrm{min}$ from 5 to $-120^{\circ} \mathrm{C}$. Subsequently, the straws were immersed into liquid nitrogen at $-196^{\circ} \mathrm{C}$. All straws were kept in liquid nitrogen for a minimum of $1 \mathrm{wk}$ before thawing.

\section{Seminal Plasma Arising from the Whole Sperm- Rich Fraction Collection and Storage}

Autologous SP arising from the whole SRF was obtained from the same sample collection used for cryopreservation. The supernatant previously obtained was centrifuged $\left(2,500 \times g\right.$ for $30 \mathrm{~min}$ at $\left.25^{\circ} \mathrm{C}\right)$, completely separated from the pellet by aspiration, submitted to vacuum filtration through disposable filters with a 0.22- $\mu$ m diameter (99150, Filtermax; TPP Techno Plastic Products AG, Trasadingen, Switzerland), and stored at $-80^{\circ} \mathrm{C}$ for further use. 


\section{Semen Thawing and a Novel Distribution into Treatments}

Two straws from each treatment (CT, CS, and CW) were thawed in a water bath at $37^{\circ} \mathrm{C}$ for $30 \mathrm{~s}$. Thawed semen was diluted to a final concentration of $5 \times 10^{6}$ spermatozoa $/ \mathrm{mL}$ in freezing extender. Additionally, 2 straws from the $\mathrm{CW}$ treatment were thawed and diluted with freezing extender supplemented with $10 \%$ autologous SP arising from the whole SRF (vol/vol; Vadnais et al., 2005) to create the treatment CWSP (post-thawed SP arising from the whole SRF addition to $\mathrm{CW}$ [CW containing $10 \%$ of autologous SP-SRF after thawing]). Thawed samples were kept in a water bath at $37^{\circ} \mathrm{C}$ until all analyses were completed.

\section{Flow Cytometry Analysis}

An aliquot from each experimental treatment was stained and analyzed at 3 incubation times: 0 (allowed to equilibrate for $5 \mathrm{~min}$ ), 60, and $120 \mathrm{~min}$. Samples for staining and flow cytometry analysis were diluted in TALP or mTALP and stained with H342 $(33 \mu \mathrm{g} / \mathrm{mL}$ in $150 \mu \mathrm{L}$ at $5 \times 10^{6}$ spermatozoa $/ \mathrm{mL}$; photomultiplier tubes [PMT] with a band pass of $450 \pm 10 \mathrm{~nm}$ ) for $10 \mathrm{~min}$ at $37^{\circ} \mathrm{C}$. Hoechst 33342 is a dye-permeable membrane cell to stain the DNA of the sperm cells. Therefore, particles with the same scatter properties as spermatozoa were not counted as spermatozoa (de Andrade et al., 2012). After addition of the fluorescent dyes for each analysis, the incubated semen samples were analyzed in a BD FACSAria flow cytometer (Becton, Dickinson and Company, San Jose, CA) controlled by BD FACSDiva 6.0 software (Becton, Dickinson and Company). The spectral overlap of spermatozoa staining in the flow cytometer analyses was compensated whenever necessary. The samples were processed through the instrument at an acquisition rate of approximately 600 to 1,000 events/s, acquiring 10,000 cells per analysis. The cells were simultaneously excited by an argon laser at $488 \mathrm{~nm}$ and by a near-UV laser at $375 \mathrm{~nm}$.

\section{Stability Evaluation (Capacitation) of the Plasma Membrane}

Hoechst 33342-incubated samples were subsequently stained with $0.5 \mu \mathrm{L}$ of Yo-Pro-1 (7.5 $\mu M$; PMT with a long pass of 502 and a band pass of 530 $\pm 15 \mathrm{~nm}$ ). Yo-Pro-1 is a dye impermeable to the intact plasma membrane, but it can cross the damaged plasma membrane. After $20 \mathrm{~min}$ of incubation, $0.5 \mu \mathrm{L}$ of M540 $(810 \mu M)$ fluorescent probe was added followed by incubation for $70 \mathrm{~s}$ (Flesch et al., 1999; Rathi et al., 2001). The hydrophobic dye M540 is able to monitor the level of lipid disorder of the plasma membrane lipid bilayer (Harrison et al., 1996). Samples were then diluted in $150 \mu \mathrm{L}$ of mTALP and were analyzed by flow cytometry. Viable cells (Yo-Pro-1 negative cells) were analyzed by M540 fluorescence captured in the long pass $(595 \mathrm{~nm})$ and band pass $(610 \pm 20 \mathrm{~nm})$ regions.

\section{Evaluation of Lipid Peroxidation in Sperm Cells with Intact Plasma Membranes}

Aliquots of H342-incubated samples were stained with $0.5 \mu \mathrm{L}$ of C11-BODIPY ${ }^{581 / 591}$ probe $(1 \mathrm{mg} / \mathrm{mL})$ for $30 \mathrm{~min}$ at $37^{\circ} \mathrm{C}$. This probe incorporates into biological membranes and responds to free radical attack (peroxyl and alkoxyl) with an increase in green fluorescence emission, which can be readily monitored and quantified by flow cytometry (Neild et al., 2005). After the incubation period, $3 \mu \mathrm{L}$ of propidium iodide (PI; $0.5 \mathrm{mg} / \mathrm{mL}$; PMT with a long pass of $655 \mathrm{~nm}$ and band pass of $695 \pm 20 \mathrm{~nm}$ ) was added to exclude cells with a damaged plasma membrane from flow cytometer analysis. After incubation for $5 \mathrm{~min}$ at $37^{\circ} \mathrm{C}$, sperm were diluted in TALP to $2.5 \times 10^{6}$ spermatozoa $/ \mathrm{mL}$ and were analyzed by flow cytometry. The mean intensity of fluorescence emission (arbitrary units), captured by the PMT with a long pass of 502 and a band pass of $530 \pm 15 \mathrm{~nm}$, was analyzed for the viable sperm (PI negative) and C11-BODIPY ${ }^{581 / 591}$-positive sperm.

\section{Simultaneous Assessment of Plasma and Acrosomal Membranes}

Propidium iodide $(10 \mu \mathrm{g} / \mathrm{mL})$ and Pisum sativum agglutinin conjugated to fluorescein isothiocyanate (FITC-PSA; $7 \mu \mathrm{g} / \mathrm{mL}$; PMT with a long pass of 502 and a band pass of $530 \pm 15 \mathrm{~nm}$ ) were used added to $147 \mu \mathrm{L}$ of $\mathrm{H} 342$-incubated samples. The purpose of these probes was to stain the cells with damaged plasma membranes (PI positive; de Andrade et al., 2007) as well as those that had undergone the acrosome reaction (FITC-PSA positive). After 10 min of incubation at $37^{\circ} \mathrm{C}$, the samples were diluted by the addition of $150 \mu \mathrm{L}$ of TALP and were analyzed by flow cytometry.

\section{Statistical Analysis}

Data were analyzed using the MIXED procedure of SAS software (SAS Inst. Inc., Cary, NC), according to a randomized block design with repeated measures. The treatments were considered the fixed variables, and each boar (considered a block) was considered the random variable. Treatment effects were evaluated using contrasts: contrast $1(\mathrm{C} 1=\mathrm{CT}$ vs. $\mathrm{CS})$, the effect of centrifugation; contrast $2(\mathrm{C} 2=\mathrm{CW}$ vs. $\mathrm{CT}$ and 
CWSP), the effect of absence of SP-SRF regardless of $\mathrm{SP}$ addition time; and contrast $3(\mathrm{C} 3=\mathrm{CW}$ vs. $\mathrm{CWSP})$, the effect of SP-SRF addition on post-thawed media in reverse absence effect. Time effects were evaluated using the Tukey-Kramer SAS MIXED procedure. Effects were considered significant when $P<0.05$, and all results were expressed as the means \pm SEM.

\section{RESULTS}

Time $\times$ treatment had no interaction $(P>0.05)$ for all variables; therefore, we studied the effects of the time and treatment separately.

\section{Plasma Membrane Phospholipid Disorder (Capacitation)}

The prefreezing centrifugation process decreased $(P<0.05)$ fluorescence emission of M540. Nevertheless, absence of SP -SRF increased $(P<0.05)$ the plasma membrane phospholipid disorder. The addition of SP from the whole SRF after thawing or its maintenance during cryopreservation decreased $(P<0.05)$ the plasma membrane phospholipid disorder (Fig. 1A). However, no change in the plasma lipid disorder was observed during $120 \mathrm{~min}$ of incubation $(P>0.05)$.

\section{Sperm Plasma Membrane Lipid Peroxidation}

The fluorescence emission of C11-BODIPY $581 / 591$ decreased $(P<0.05)$ in the centrifuged samples. However, the absence of SP-SRF had no influence $(P>$ 0.05 ) on the effect of oxygen reactive species in the plasma membrane phospholipids. The addition of $10 \%$ of SP from the whole SRF did not reduce $(P>0.05)$ lipid peroxidation of the sperm with plasma membrane integrity (PI negative; Fig. 1B). Sperm lipid peroxidation remained similar $(P>0.05)$ during the initial 60 min of incubation but was increased $(P<0.05)$ by 120 min of incubation compared with peroxidation observed during the first $5 \mathrm{~min}$ of incubation (Table 1).

\section{Plasma and Acrosomal Membrane Integrity}

The process of centrifugation prefreezing was not decrease the plasma and acrosomal membrane integrity (sperm with intact acrosomal and plasma membrane [AIPI; $P>0.05$ ], sperm with intact acrosomal and damaged plasma membrane [AIPD; $P>0.05$ ], sperm with reacted acrosomal membrane and intact plasma membrane [ARPI; $P>0.05]$, and sperm with reacted acrosomal membrane and damaged plasma membrane [ARPD; $P>$ 0.05]; Fig. 1C). The addition of SP from the whole SRF to the thawing medium increased $(P<0.05)$ the number

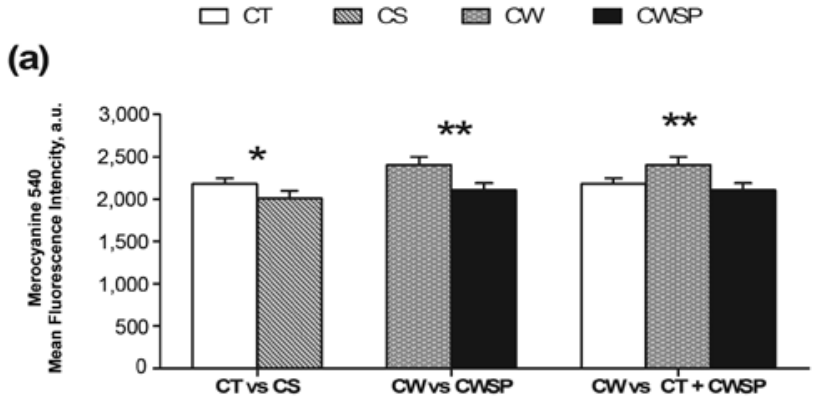

(b)
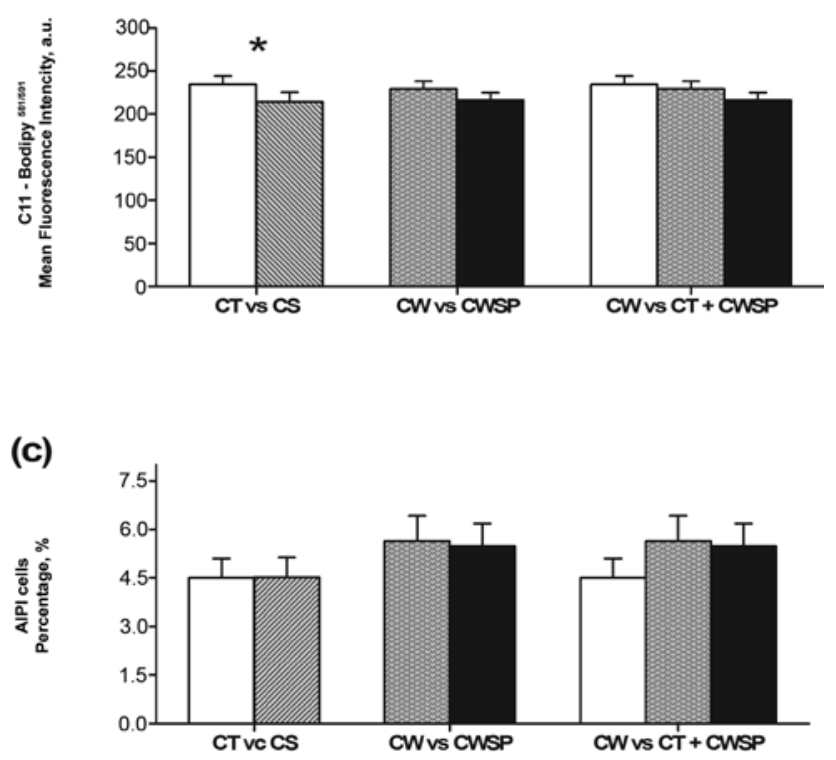

Figure 1. Whole sperm-rich fraction seminal plasma effects on fluidity, peroxidation, and integrity of sperm membranes. (A) Mean \pm SE of membrane disorder. (B) Mean $\pm \mathrm{SE}$ of membrane peroxidation. (C) Mean $\pm \mathrm{SE}$ of spermatozoa with simultaneous plasma and acrosome integrity. CT $=$ control; $\mathrm{CS}=$ centrifuged and suspended in autologous seminal plasma (pellet was gently mixed in its own supernatant, without seminal plasma withdraw); $\mathrm{CW}=$ centrifuged with withdrawn seminal plasma; CWSP = post-thawed seminal plasma arising from the whole sperm-rich fraction addition to $\mathrm{CW}$ (CW containing 10\% autologous seminal plasma); $\mathrm{AIM}=$ sperm with intact acrosomal and plasma membrane; a.u. = arbitrary units; $\mathrm{C} 1=$ centrifugation effect $(\mathrm{C} 1=\mathrm{CT}$ vs. $\mathrm{CS}) ; \mathrm{C} 2=$ effect of absence of seminal plasma regardless seminal plasma addition time $(\mathrm{C} 2=\mathrm{CW}$ vs. $\mathrm{CT}$ and CWSP); $\mathrm{C} 3$ = effect of seminal plasma addition on post-thawed media in reverse absence effect $(\mathrm{C} 3=\mathrm{CW}$ vs. CWSP). Asterisks indicate differences between individual means $(* P<0.05 ; * * P<0.01)$.

of ARPI, but this increase was not observed in the other cell groups (AIPI, $>0.05$; IPD, $P>0.05$; and ARPD, $P>$ 0.05 ; Fig. 1C). The absence of SP -SRF during the process of freezing/thawing did not alter the acrosomal and plasma membrane integrity (AIPI, $P>0.05$; AIPD; $P>$ 0.05 ; ARPI, $P>0.05$; and ARPD, $P>0.05)$. However, the percentage of AIPI $(P<0.05)$ and AIPD $(P<0.05)$ cells were reduced and the percentage of ARPD cells was increased $(P<0.05)$ during the first $60 \mathrm{~min}$ of incubation; ARPI cells remained stable $(P>0.05)$ during this period. When the incubation time was extended to $120 \mathrm{~min}$, the percentage of AIPI $(P>0.05)$ and AIPD $(P>0.05)$ cells remained stable when compared with the 60-min incuba- 
Table 1. Effect of time on acrosomal and plasma membrane integrity and membrane peroxidation (mean $\pm \mathrm{SE}$ of periods of 5,60 , and 120 min of incubation at $37^{\circ} \mathrm{C}$ )

\begin{tabular}{|c|c|c|c|c|}
\hline \multirow[b]{2}{*}{ Sperm membrane characteristics } & \multicolumn{3}{|c|}{ Period, min } & \multirow[b]{2}{*}{$P$-value } \\
\hline & 5 & 60 & 120 & \\
\hline Acrosome and plasma membrane intact, $\%$ & $9.02 \pm 0.7^{\mathrm{a}}$ & $3.68 \pm 0.36^{\mathrm{b}}$ & $2.03 \pm 0.23^{b}$ & $<0.0001$ \\
\hline Acrosome intact and plasma membrane damage, $\%$ & $5.53 \pm 0.4^{\mathrm{a}}$ & $4.14 \pm 0.2^{b}$ & $3.36 \pm 0.16^{\mathrm{b}}$ & $<0.0001$ \\
\hline Acrosome reacted and plasma membrane intact, \% & $4.11 \pm 0.52^{\mathrm{a}}$ & $3.08 \pm 0.35^{\mathrm{a}}$ & $1.71 \pm 0.25^{\mathrm{b}}$ & $<0.0001$ \\
\hline Acrosome and plasma membrane damage, $\%$ & $81.89 \pm 1.03^{\mathrm{a}}$ & $89.08 \pm 0.6^{\mathrm{b}}$ & $93.34 \pm 0.43^{\mathrm{c}}$ & $<0.0001$ \\
\hline Membrane peroxidation, a.u. ${ }^{1}$ & $243.7 \pm 8.8^{\mathrm{a}}$ & $223.7 \pm 8.55^{\mathrm{a}, \mathrm{b}}$ & $203.7 \pm 7.56^{\mathrm{b}}$ & 0.0028 \\
\hline
\end{tabular}

${ }^{\mathrm{a}-\mathrm{c}}$ Different letters in the same row indicate a significant difference between individual means $(P<0.05)$.

$1_{\text {a.u. }}=$ arbitrary units.

tion period. A decrease in ARPI spermatozoa $(P<0.05)$ and an increase in ARPD spermatozoa $(P<0.05)$ during the 60 and 120 min incubation are shown in Table 1.

\section{DISCUSSION}

The SRF is a complex mixture composed mainly of epididymal fluid and a low proportion of prostatic and vesicular fluids (Rodríguez-Martínez et al., 2011). In boars, the majority of SP proteins (90\%) belong to the spermadhesin family, which can be categorized according to its ability to bind heparin (AQN-1, AQN3 , and AWN, which are heparin-binding proteins, and PSP-I and PSP-II, which are non-heparin-binding proteins; Caballero et al., 2008). The heparin-binding protein monomers are able to bind directly to the plasma membrane (Caballero et al., 2012), preventing not only in vitro capacitation but also capacitation-like changes induced by cold shock (Vadnais and Roberts, 2010). Furthermore, heterodimers PSP-I prevent premature capacitation (Kwok et al., 1993). These data support our findings that SP, either maintained during cryopreservation or added after thawing, increases plasma membrane stability, as shown by M540 when evaluated by flow cytometry. Another class of proteins, type 2 fibronectins (FN-2), is responsible for stabilizing the sperm membrane by binding to phosphatidylcholine (Villemure et al., 2003; Lusignan et al., 2007). Therefore, the removal of SP shortly after ejaculation decreases the interaction of spermatozoa and those proteins, which results in post-thawed membrane destabilization, as was found in the current study.

Seminal plasma removal is a widespread procedure in traditional boar semen cryopreservation protocols; this removal is essential to increase sperm concentration (Gómez-Fernández et al., 2013), allowing for semen storage in 0.25 - and 0.5 -mL straws. In contrast, SP represents the seminal fraction with the greatest antioxidant capacity; its removal renders sperm cells vulnerable to lipoperoxidation due to depletion of factors responsible for the uptake of reactive oxygen species (ROS; Gómez-Fernández et al., 2013; Marshburn et al., 2014). However, our results did not demonstrate this detrimental effect. In the current study, lipid membrane peroxidation occurred similarly in samples with and without SP. As mentioned above, the absence of SP increased plasma lipid disorder. This membrane alteration could, therefore, be caused by the reaction of ROS (de Lamirande et al., 1998; O'Flaherty et al., 1999). These results lead to the conclusion that the ROS concentration required to initiate a physiological reaction is minimal when compared with those needed to trigger lipoperoxidation. Similar results were found in equine species (de Andrade et al., 2012), in which the addition of SP decreased plasma membrane instability and tyrosine phosphorylation but was not able to prevent the ROS effects triggering lipid peroxidation.

The sperm plasma membrane is the main site of damage caused by the process of cryopreservation, which is a result of osmotic stress, cold shock, or intra- and extracellular ice formation (Mazur, 1984). Boar sperm cells are notoriously sensitive to freezing, which results in a decrease in sperm motility and in a proportional increase of plasma and acrosomal membrane damage (Saravia et al., 2005). Several studies have reported that these effects can be reduced by adding SP from whole ejaculates to the thawing medium, as performed in equine (de Andrade et al., 2011), human (Han et al., 1990), and swine species (Hernández et al., 2007). However, these beneficial effects were not seen in this study; the addition of SP from the SRF did not increase the percentage of AIPI cells. Even when we considered solely the presence or absence of SP, there were no positive effects on the plasma and acrosomal membrane integrity of the post-thawed swine spermatozoa.

Spermatozoa function and metabolism are both controlled by essential components of SP (Maxwell and Johnson, 1999). However, the presence of SP during cryopreservation is deleterious to sperm cell physiology. Nevertheless, its presence is essential after thawing (Okazaki et al., 2009). For the acrosomal 
reaction, SP shows its effects through a number of molecular systems, such as the proteasome-like vesicles present in swine ejaculate, which are one of many other factors responsible for triggering the acrosomal reaction (Siciliano et al., 2008). Therefore, these fundamental processes might not work properly in the absence of SP, which was suggested by our results regarding the acrosomal reaction.

Therefore, this work expands the scientific perspectives for the study of SP in relation to their effects on sperm membrane integrity, fluidity, and peroxidation. In conclusion, 1) the addition of SP from the whole SRF to post-thawed boar semen reverses the increase in sperm lipid disorder caused by SP withdrawal, which may decrease the cryocapacitation and improve boar semen cryopreservation, but 2) the addition or maintenance of SP from the whole SRF did not minimize the cryopreservation damage to sperm membrane integrity and peroxidation, although these results are found in the literature, and 3) this study needs to be carefully interpreted because the effects of the absence/presence of SP could be mistaken for the centrifugation effects. However, our study showed that even though centrifugation has an effect on the cryopreserved spermatozoa, the presence of SP from the whole SRF on the postthawed media is crucial to boar spermatozoa.

\section{LITERATURE CITED}

Bavister, B. D., M. L. Leibfried, and G. Lieberman. 1983. Development of preimplantation embryos of the golden hamster in a defined culture medium. Biol Reprod. 28:235-247.

Caballero, I., I. Parrilla, C. Almiñana, D. Del Olmo, J. Roca, E. Martínez, and J. Vázquez. 2012. Seminal plasma proteins as modulators of the sperm function and their application in sperm biotechnologies. Reprod. Domest. Anim. 47:12-21. doi:10.1111/j.14390531.2012.02028.x

Caballero, I., J. M. Vazquez, E. M. García, I. Parrilla, J. Roca, J. J. Calvete, L. Sanz, and E. A. Martínez. 2008. Major proteins of boar seminal plasma as a tool for biotechnological preservation of spermatozoa. Theriogenology 70:1352-1355. doi:10.1016/j. theriogenology.2008.07.013

de Andrade, A. F. C., R. P. de Arruda, E. C. C. Celeghini, J. Nascimento, S. M. M. K. Martins, C. F. Raphael, and A. S. Moretti. 2007. Fluorescent stain method for the simultaneous determination of mitochondrial potential and integrity of plasma and acrosomal membranes in boar sperm. Reprod. Domest. Anim. 42:190-194. doi:10.1111/j.1439-0531.2006.00751.x

de Andrade, A. F. C., F. G. Zaffalon, E. C. C. Celeghini, J. Nascimento, F. F. Bressan, S. M. M. K. Martins, and R. P. de Arruda. 2012. Post-thaw addition of seminal plasma reduces tyrosine phosphorylation on the surface of cryopreserved equine sperm, but does not reduce lipid peroxidation. Theriogenology 77:1866-1872.e1-3. doi:10.1016/j.theriogenology.2012.01.003 de Andrade, A. F. C., F. G. Zaffalon, E. C. C. Celeghini, J. Nascimento, O. F. B. Tarragó, S. M. M. K. Martins, M. A. Alonso, and R. P. Arruda. 2011. Addition of seminal plasma to postthawing equine semen: What is the effect on sperm cell viability? Reprod. Domest. Anim. 46:682-686. doi:10.1111/j.14390531.2010.01729.x

de Lamirande, E., C. Tasi, A. Harakat, and C. Gagnon. 1998. Involvement of reactive oxygen species in human sperm acrosome reaction induced by A23187, lysophosphatidylcholine, and biological fluid ultrafiltrates. J. Androl. 19:585-594.

Fernández-Gago, R., J. C. Domínguez, and F. Martínez-Pastor. 2013. Seminal plasma applied post-thawing affects boar sperm physiology: A flow cytometry study. Theriogenology 80:400410. doi:10.1016/j.theriogenology.2013.05.003

Flesch, F. M., B. Colenbrander, L. M. van Golde, and B. M. Gadella. 1999. Capacitation induces tyrosine phosphorylation of proteins in the boar sperm plasma membrane. Biochem. Biophys. Res. Commun. 262:787-792. doi:10.1006/bbrc.1999.1300

García, E., J. Calvete, L. Sanz, J. Roca, E. Martínez, and J. Vázquez. 2009. Distinct effects of boar seminal plasma fractions exhibiting different protein profiles on the functionality of highly diluted boar spermatozoa. Reprod. Domest. Anim. 44:200-205. doi:10.1111/j.1439-0531.2007.01028.x

Garner, D. L., C. A. Thomas, C. G. Gravance, C. E. Marshall, J. M. Dejarnette, and C. Allen. 2001. Seminal plasma addition attenuates the dilution effect in bovine sperm. Theriogenology 56:31-40. doi:10.1016/S0093-691X(01)00540-4

Gómez-Fernández, J., E. Gómez-Izquierdo, C. Tomás, E. Mocé, and E. de Mercado. 2013. Is sperm freezability related to the post-thaw lipid peroxidation and the formation of reactive oxygen species in boars? Reprod. Domest. Anim. 48:177-182. doi:10.1111/j.1439-0531.2012.02126.x

Han, H. L., S. R. Mack, C. De Jonge, and L. J. D. Zeneveld. 1990. Inhibition of the human sperm acrosome reaction by a high molecular weight factor from human seminal plasma. Fertil. Steril. 54:1177-1179.

Harrison, R. A. P., P. J. C. Ashworth, and N. G. A. Miller. 1996. Bicarbonate $/ \mathrm{CO}_{2}$, an effector of capacitation, induces a rapid and reversible change in the lipid architecture of boar sperm plasma membranes. Mol. Reprod. Dev. 45:378-391. doi:10.1002/ (SICI)1098-2795(199611)45:3<378::AID-MRD16>3.0.CO;2-V

Hernández, M., J. Roca, J. J. Calvete, L. Sanz, T. Muiño-Blanco, J. A. Cebrián-Pérez, J. M. Vázquez, and E. A. Martínez. 2007. Cryosurvival and in vitro fertilizing capacity postthaw is improved when boar spermatozoa are frozen in the presence of seminal plasma from good freezer boars. J. Androl. 28:689-697. doi:10.2164/jandrol.107.002725

Kwok, S. C. M., M. J. Soares, J. P. McMurtry, and E. C. Yurewicz. 1993. Binding characteristics and immunolocalization of porcine seminal protein, PSP-I. Mol. Reprod. Dev. 35:244-250. doi:10.1002/ mrd.1080350305

Lusignan, M. F., A. Bergeron, M. H. Crete, C. Lazure, and P. Manjunath. 2007. Induction of epididymal boar sperm capacitation by $\mathrm{pB} 1$ and BSP-A1/-A2 proteins, members of the BSP protein family. Biol. Reprod. 76:424-432. doi:10.1095/biolreprod.106.055624

Marshburn, P. B., A. Giddings, S. Causby, M. L. Matthews, R. S. Usadi, N. Steuerwald, and B. S. Hurst. 2014. Influence of ejaculatory abstinence on seminal total antioxidant capacity and sperm membrane lipid peroxidation. Fertil. Steril. 102:705-710. doi:10.1016/j. fertnstert.2014.05.039 
Maxwell, W. M. C., and L. A. Johnson. 1999. Physiology of spermatozoa at high dilution rates: The influence of seminal plasma. Theriogenology 52:1353-1362. doi:10.1016/S0093691X(99)00222-8

Mazur, P. 1984. Freezing of living cells: Mechanisms and implications. Am. J. Physiol. Cell Physiol. 247:125-142.

Neild, D. M., J. F. Brouwers, B. Colenbrander, A. Agüero, and B. M. Gadella. 2005. Lipid peroxide formation in relation to membrane stability of fresh and frozen thawed stallion spermatozoa. Mol. Reprod. Dev. 72:230-238. doi:10.1002/mrd.20322

O’Flaherty, C. M., N. B. Beorlegui, and M. T. Beconi. 1999. Reactive oxygen species requirement for bovine sperm capacitation and acrosome reaction. Theriogenology 52:289-301. doi:10.1016/S0093691X(99)00129-6

Okazaki, T., S. Abe, S. Yoshida, and M. Shimada. 2009. Seminal plasma damages sperm during cryopreservation, but its presence during thawing improves semen quality and conception rates in boars with poor post-thaw semen quality. Theriogenology 71:491-498. doi:10.1016/j.theriogenology.2008.08.014

Pursel, V. G., L. Schulman, and L. A. Johnson. 1973. Effect of holding time on storage of boar spermatozoa at $5^{\circ} \mathrm{C}$. J. Anim. Sci. 37:785-789.

Rathi, R., B. Colenbrander, M. M. Bevers, and B. M. Gadella. 2001. Evaluation of in vitro capacitation of stallion spermatozoa. Biol. Reprod. 65:462-470. doi:10.1095/biolreprod65.2.462

Rodríguez-Martínez, H., U. Kvist, J. Ernerudh, L. Sanz, and J. J. Calvete. 2011. Seminal plasma proteins: What role do they play? Am. J. Reprod. Immunol. 66:11-22. doi:10.1111/j.16000897.2011.01033.x

Rovegno, M., W. B. Feitosa, A. M. Rocha, C. M. Mendes, J. A. Visintin, and M. E. O. D'Avila Assumpção. 2013. Assessment of post-thawed ram sperm viability after incubation with seminal plasma. Cell Tissue Bank. 14:333-339. doi:10.1007/s10561012-9317-1

Saravia, F., M. Wallgren, A. Johannisson, J. J. Calvete, L. Sanz, F. J. Peña, J. Roca, and H. Rodríguez-Martínez. 2009. Exposure to the seminal plasma of different portions of the boar ejaculate modulates the survival of spermatozoa cryopreserved in MiniFlatPacks. Theriogenology 71:662-675. doi:10.1016/j.theriogenology.2008.09.037
Saravia, F., M. Wallgren, A. Johannisson, and H. Rodriguez-Martinez. 2007. Controlled cooling during semen cryopreservation does not induce capacitation of spermatozoa from two portions of the boar ejaculate. Int. J. Androl. 30:485-499. doi:10.1111/j.13652605.2006.00741.x

Saravia, F., M. Wallgren, S. Nagy, A. Johannisson, and H. Rodríguez-Martínez. 2005. Deep freezing of concentrated boar semen for intra-uterine insemination: Effects on sperm viability. Theriogenology 63:1320-1333. doi:10.1016/j.theriogenology.2004.06.012

Siciliano, L., V. Marcianò, and A. Carpino. 2008. Prostasome-like vesicles stimulate acrosome reaction of pig spermatozoa. Reprod. Biol. Endocrinol. 6:5. doi:10.1186/1477-7827-6-5

Siqueira, A. P., M. Wallgren, M. S. Hossain, A. Johannisson, L. Sanz, J. J. Calvete, and H. Rodríguez-Martínez. 2011. Quality of boar spermatozoa from the sperm-peak portion of the ejaculate after simplified freezing in MiniFlatpacks compared to the remaining spermatozoa of the sperm-rich fraction. Theriogenology 75:1175-1184. doi:10.1016/j.theriogenology.2010.11.024

Vadnais, M. L., R. N. Kirkwood, D. J. Specher, and K. Chou. 2005. Effects of extender, incubation temperature, and added seminal plasma on capacitation of cryopreserved, thawed boar sperm as determined by chlortetracycline staining. Anim. Reprod. Sci. 90:347-354. doi:10.1016/j.anireprosci.2005.02.007

Vadnais, M. L., and K. P. Roberts. 2010. Seminal plasma proteins inhibit in vitro- and cooling-induced capacitation in boar spermatozoa. Reprod. Fertil. Dev. 22:893-900. doi:10.1071/RD09274

Villemure, M., C. Lazure, and P. Manjunath. 2003. Isolation and characterization of gelatin-binding proteins from goat seminal plasma. Reprod. Biol. Endocrinol. 1:39-48. doi:10.1186/14777827-1-39

Zasiadczyk, L., L. Fraser, W. Kordan, and K. Wasilewska. 2015. Individual and seasonal variations in the quality of fractionated boar ejaculates. Theriogenology 83:1287-1303. doi:10.1016/j.theriogenology.2015.01.015 